\title{
BLV-CoCoMo-qPCR-3: Improved BLV-CoCoMo-qPCR for Bovine Leukemia Virus Detection by Mixing Probes Targeting all BLV Variants
}

Liushiqi Borjigin

Nakamura Laboratory, Baton Zone Program, RIKEN Cluster for Science, Technology and Innovation Hub, Wako, Saitama

Shuji Yoneyama

Central Livestock Hygiene Service Center of Tochigi Prefecture, Utsunomiya, Tochigi

Susumu Saito

Nakamura Laboratory, Baton Zone Program, RIKEN Cluster for Science, Technology and Innovation Hub, Wako, Saitama

Polat Meripet

Nakamura Laboratory, Baton Zone Program, RIKEN Cluster for Science, Technology and Innovation Hub, Wako, Saitama

Michihito Inokuma

Chuo Livestock Hygiene Service Center of Chiba Prefecture, Chiba

Yasuo Shinozaki

Nanbu Livestock Hygiene Service Center of Chiba Prefecture, Kamogawa, Chiba

Naoko Tanaka

Nanbu Livestock Hygiene Service Center of Chiba Prefecture, Kamogawa, Chiba

Risa Yamanaka

Kumagaya Livestock Hygiene Service Center of Saitama Prefecture, Kumagaya, Saitama

Anna Yasui

Kumagaya Livestock Hygiene Service Center of Saitama, Kumagaya, Saitama

Munehito Mimura

Kumagaya Livestock Hygiene Service Center of Saitama Prefecture, Kumagaya, Saitama

Hironobu Murakami

Laboratory of Animal Health $₫$, School of Veterinary Medicine, Azabu University, Sagamihara, Kanagawa

Shin-nosuke Takeshima

Department of Food and Nutrition, Jumonji University, Niiza Saitama

Yoko Aida ( $\nabla$ aida@riken.jp )

RIKEN https://orcid.org/0000-0001-7400-3587

\section{Research}

Keywords: Bovine leukemia virus, Real-time PCR, BLV-CoCoMo-qPCR, Cattle, Proviral load, mixing probe, LTR

Posted Date: November 2nd, 2020

DOI: https://doi.org/10.21203/rs.3.rs-99137/v1

License: (1) This work is licensed under a Creative Commons Attribution 4.0 International License. Read Full License 


\section{Abstract}

Background: The bovine leukemia virus (BLV) is the causative agent of enzootic bovine leukosis, the most common neoplastic disease in cattle. The proviral load (PVL) is an important index for estimating disease progression. Previously, we developed a quantitative real-time PCR (qPCR) assay to measure the PVL of BLV using coordination of common motif (CoCoMo) degenerate primers that can amplify all known BLV strains. However, mutations, which potentially affect the detection ability, have been recently reported in the probe sequences of the long terminal regions (LTRs) of the BLV-CoCoMo-qPCR-2 assay. Here, we developed a new strategy to overcome these newly generated mutations located in the probe regions of this assay.

Methods: We collected genomic DNA from 887 cows from 27 BLV-positive farms, using a nationwide survey conducted in 2011 and 2017 in Japan. BLV variants were investigated by quantifying the provirus using BLV-CoCoMo-qPCR-2 targeting the BLV LTR gene and the TaKaRa Cycleave PCR system targeting the BLV tax gene. Additionally, we sequenced the partial BLV LTR gene. The modified probes were designed to completely match the three BLV variants identified here, and the modified assay was established using mixed probes.

Results: We found four single mutations within the probe region of the original BLV-CoCoMo-qPCR-2 assay, three of which negatively affected its sensitivity. Furthermore, we examined the optimum ratio of the concentration to be mixed with the wild type and three new BLV TaqMan probes were designed here using genomic DNAs extracted from cattle infected with the wild-type BLV strain and cattle infected with variants. Hence, we successfully established an improved BLV-CoCoMo-qPCR-3 assay that uses mixed probes corresponding to all three BLV variants.

Conclusions: To overcome the loss of assay sensitivity due to newly emerging variants, we have established the BLV-CoCoMo-qPCR-3 assay that could amplify all BLV strains using newly designed mixed probes in addition to degenerate primers that were previously designed in our original assay. Our proposed method maintained the original sensitivity and reproducibility and can detect all mutant strains; thus, it is a useful tool to prevent the spread of BLV infections, especially those caused by newly emerging variants.

\section{Background}

Bovine leukemia virus (BLV) belongs to the family Retroviridae (genus Deltaretrovirus), together with human T-leukemia virus types 1 and 2 (HTLV-1 and - 2 ), and causes enzootic bovine leucosis (EBL), the most common neoplastic disease affecting cattle worldwide [1]. Approximately $70 \%$ of BLV-infected cattle are asymptomatic, a stage designated as aleukemic, whereas approximately $25-30 \%$ and $1-5 \%$ of BLV-infected cattle develop persistent lymphocytosis and B cell lymphoma after several years of latency, respectively [1]. BLV infection commonly affects the cattle industry worldwide and causes significant economic losses due to decreased milk yield [2-5] and daily gain [6], decreased immunity levels [7], and reduced reproductive capacity [6] and longevity [2, 8].

Because BLV can cause silent infections in cattle at all disease stages, detecting the BLV provirus, the retroviral genome integrated into the host genome [9, 10], even in the absence of detectable BLV antibodies [11] is crucial for diagnosing BLV infection [10, 12-16]. Particularly, the BLV proviral load (PVL) correlates strongly with not only the BLV infection capacity (as assessed by syncytium formation) $[17,18]$ but also EBL progression [19, 20]. For example, BLV normally transmits horizontally and vertically, and cattle with high PVLs are considered a major risk factors for infection [11, 21-24] and for the progression of EBL $[11,17,25]$. Indeed, previous reports have posited that as determined by BLV-CoCoMo-quantitative polymerase chain reaction (qPCR)-2 [26], BLV provirus may be detected in the milk, nasal mucus, and saliva of dairy cattle with PVLs $>10,000,14,000$, and 18,000 copies per $10^{5}$ cells in blood samples, respectively $[23,24]$. These infected cattle may pose an increased risk of BLV transmission when in direct contact with healthy cattle. In contrast, cattle with low PVLs are less likely to transmit BLV to other cattle [27]. Thus, PVL is a useful index for estimating the risk of transmission.

We previously developed a BLV-CoCoMo-qPCR assay using coordination of common motifs (CoCoMo) degenerate primers to amplify both known and novel BLV variants and an internal BLV TaqMan probe to enhance the specificity and sensitivity of the assay targeting the long terminal repeat (LTR) gene of BLV $[17,28]$. This LTR region is present at two copies per provirus, contributing to the assay's improved sensitivity and the highly sensitive detection of BLV variants from different countries [17]. In addition, to normalize the levels of BLV to those of viral genomic DNA, this technique amplifies bovine leukocyte antigen (BoLA)-DRA--a single-copy host gene--in parallel with the viral genomic DNA. This allows for adjusting variations in amplification efficiency between samples. Recently, a modified version CoCoMo-qPCR-2, which uses optimized degenerate primers and the construction of an optimal standard curve, was improved in 2015 [26]. This modified version has been widely used by BLV researchers and veterinarians [11, 13-16, 18, 24, 29-38] due to its quantitative nature and high specificity, sensitivity, and reproducibility.

In general, increasing variations in the genomic sequences of microorganisms are the main reason why they escape host immune responses. Furthermore, they can avoid the current detection assays that target the viral gene by undergoing mutation. Hence, it may be necessary to improve the detection methods for mutated viruses from time to time. Recently, the three types of mutations have been reported in the nucleobase of BLV LTR regions [15, 39, 40]. However, if these mutations are present in the probe region in the LTRs of the BLV-CoCoMo-qPCR-2 assay, the assay may not be able to detect all BLV strains. In this study, first of all, we investigated the mutant BLV strains by using genomic DNA of 887 cattle from 27 farms in 13 prefectures in Japan. Moreover, we constructed three new probes to amplify variants with single substitutions. Finally, we developed a modified version of the BLV-CoCoMo-qPCR assay--the BLV-CoCoMo-qPCR-3 assay--that can detect all BLV mutant strains using a mix of wild type and three new BLV TaqMan probes.

\section{Methods}

\section{Sample collection and genomic DNA extraction}

In 2011 and 2017, ethylenediaminetetraacetic acid-treated peripheral blood samples were corrected from a total of 887 cattle from 27 BLV-positive farms in 13 prefectures of Japan: 633 Holstein, 242 Japanese Black, 10 Jersey, and 2 Brown Swiss (Table 1).

Page 2/15 
Genomic DNA was extracted using the Wizard Genomic DNA Purification Kit (Promega Corporation, Tokyo, Japan), according to the manufacturer instructions.

\section{Quantification of BLV provirus using real-time PCR targeting the BLV LTR gene}

BLV PVLs were quantified using BLV-CoCoMo-qPCR-2 (RIKEN Genesis, Kanagawa, Japan) with THUNDERBIRD Probe qPCR Mix (Toyobo, Tokyo, Japan), as described previously $[17,26,28]$. In brief, a 183 bp sequence of the BLV LTR gene was amplified using the degenerate primer set "CoCoMo-FRW and CoCoMoREV" and detected with a 15 bp 6-carboxyfluorescein (FAM)-labeled LTR probe. As the internal control, the BoLA-DRA gene was amplified using the primer set "DRA-F and DRA-R" and detected with the FAM-labeled DRA probe. Finally, the PVL was calculated using the following formula: (number of BLV LTR copies/number of BoLA-DRA copies) $\times 10^{5}$ cells.

\section{Quantification of BLV provirus using real-time PCR targeting BLV tax}

BLV PVL was quantified using the Cycleave PCR system (TaKaRa Bio, Inc., Tokyo, Japan) on the 7500 FAST Real-time PCR System (Life Technologies). Briefly, BLV tax was amplified and detected with the FAM-labeled Cycleave probe using the Cycleave PCR BLV detection kit (TaKaRa Bio, Inc., Otsu, Japan). Finally, the number of copies of provirus per $100 \mathrm{ng}$ of genomic DNA was calculated.

\section{PCR amplification and sequencing of the partial BLV LTR gene}

We selected genomic DNA samples that were negative by the BLV-CoCoMo-qPCR assay but positive by the TaKaRa Cycleave PCR assay and sequenced their LTR genes. In brief, the BLV LTR gene was amplified using single-step PCR with the CoMo-t1F (5囚-ACGTCAGCTGCCAGAAAAGCTG-3囚) and CoMo-t1R (5囚AGCCAGACGCCCTTGGAGCGCG-3囚) primer set. Then, the amplified products were sequenced using the ABI PRISM Big Dye Terminator $v 1.1$ Ready Reaction Cycle Sequencing Kit (Applied Biosystems, Foster City, CA) and the CoMo-t2F (5囚-CTGGTGACGGCAGCTGGTGGC-3区) and CoMo-t3R (5囚TAGAGCTCGCGGTGGTCTCAG-3区) primer set. Sequencing data were analyzed using GENETYX version 10 (Genetyx Corporation, Tokyo, Japan).

\section{Designing new probes and establishing BLV-CoCoMo-qPCR-3}

By sequencing the LTR regions as described above, three types of BLV TaqMan probes (p242, p246, and p253) were newly constructed based on the three mutations at codon 242,246 , and 253 , respectively, in the probe region of BLV-CoCoMo-qPCR-2 corresponding to the nucleotide positions $240-254$ of the whole BLV genomic sequence recorded in GenBank (accession no. EF600696) [41]. These probes were labeled with a fluorescein amidite (FAM) dye, nonfluorescent quencher (NFQ), and minor groove binder (MGB) probe for enhancing the probe melting temperature. The FAM-LTR probes used were follows: $p$

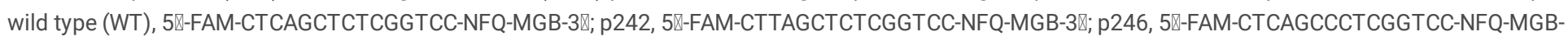
3区; and p253, 5囚-FAM-CTCAGCTCTCGGTTC-NFQ-MGB-3区.

To establish the BLV-CoCoMo-qPCR-3 assay, the ratio of wild type to mutant type probes (pWT:p242:p246:p253) was examined. Briefly, the probes were mixed at ratios (in ng) of 200:33:33:33, 150:50:50:50, 100:67:67:67, and 75:75:75:75, and the PVLs in cattle blood genomic DNA samples were quantified to verify the optimal ratio.

\section{Results}

Identification of four single substitutions between residue 240 and 254 in BLV LTR by comparison of BLV-CoCoMo-qPCR-2 targeting the BLV LTR gene and the TaKaRa Cycleave PCR system targeting the BLV tax gene

The BLV genome is highly conserved and has minor genetic variations $[15,42,43]$. However, some substitutions were recently reported to be present in the LTR region of BLV $[15,40,44]$. Therefore, to determine whether these substitutions were located in the probe region of BLV-CoCoMo-qPCR-2 corresponding to the nucleotide positions 240-254 of the LTR regions of BLV, we first collected 2115 BLV nucleotide sequences from GenBank (on 14th January, 2020), and 206 LTR sequences were selected according to GenBank annotations. As shown in Fig. 1a, comparison of the 14 LTR sequences with the sequence of the FLK-BLV strain pBLV913 [41] revealed three mutations at codons $246(T \rightarrow C), 252(T \rightarrow C)$, and $253(C \rightarrow T)$ located in the probe region in the LTR of the BLV-CoCoMoqPCR-2 assay $[15,39,40]$.

Next, to investigate whether these substitutions were really detected in BLV-positive farms in Japan, we screened for genomic DNA samples that were negative according to BLV-CoCoMo-qPCR targeting the BLV LTR gene but positive according to TaKaRa Cycleave PCR targeting the BLV tax gene and sequenced their LTR genes. BLV-CoCoMo-qPCR-2 and the TaKaRa Cycleave PCR assay were performed using the same genomic DNA samples obtained from a total of 887 cattle (Table 1): 590 (66.5\%) and 575 (64.8\%) out of 887 cattle were positive as per BLV-CoCoMo-qPCR-2 and the TaKaRa Cycleave PCR assay, respectively. As shown in Table 2, 557 samples were positive according to both BLV-CoCoMo-qPCR-2 and the TaKaRa Cycleave PCR assay (+/+), 11 were negative according to BLV-CoCoMo-qPCR-2 but positive according to the TaKaRa Cycleave PCR assay (-/+), 33 were positive according to BLV-CoCoMo-qPCR-2 but negative according to the TaKaRa Cycleave PCR system assay (+/-), and 286 were negative according to both BLV-CoCoMo-qPCR-2 and the TaKaRa Cycleave PCR assay (-/-). Thus, according to the results obtained via both methods, we selected the 11 samples which were negative according to BLV-CoCoMo-qPCR-2 but positive according to the TaKaRa Cycleave PCR assay, as targets for the sequencing test.

Furthermore, we amplified the genomic DNA extracted from those 11 samples and sequenced the partial BLV LTR gene corresponding to nucleotide positions 57-437 of the whole BLV genomic sequence [41]. We identified three types of single substitution at codons 242,246 , and 253 in the probe region of BLVCoCoMo-qPCR-2 (Fig. 1b)--namely, at codon 242 (C $\rightarrow$ T) for samples M17, M18, and M19; at codon $246(T \rightarrow C)$ for samples Y14, Y20, K4, K16, K25, T2 and $\mathrm{Cb} 15$; and at codon $253(\mathrm{C} \rightarrow \mathrm{T})$ for sample K14. Interestingly, two types of mutations at codon $246(\mathrm{~T} \rightarrow \mathrm{C})$ and at codon $253(\mathrm{C} \rightarrow \mathrm{T})$ are fitted to mutations that 
have already been registered in NCBI (Fig. 1a), whereas one type of substitution at codon $242(\mathrm{C} \rightarrow \mathrm{T})$ was not registered in NCBI. These three mutant strains were present at a rate of $1.2 \%$ (11 out of 887 samples). By contrast, substitution at codon $252(T \rightarrow C)$, which was registered in NCBI as sample pvAN003 (Fig. 1a), could not be detected in this nationwide survey conducted in 2011 and 2017. Fortunately, we could detect the mutation at the same site in a cow sample that was collected from the Tochigi prefecture in another survey conducted in 2009.

\section{Amplification curves of BLV-CoCoMo-qPCR-2 using 12 cattle DNAs with the four types of single substitutions at codons 242, 246, 252, and 253}

We first investigated the amplification curves of 12 genomic DNAs with four types of single substitutions at codons $242,246,252$, and 253 , which were identified in this nationwide survey, and 252 which was identified in another nationwide survey using BLV-CoCoMo-qPCR-2 (Fig. 2a and b). The amplification curves of the 11 mutants using a wild type probe (pWT)--such as Y14, Y20, K4, K16, K25, T2 and Cb15 for the mutation at codon 246 (T $\rightarrow$ C) (Fig. $2 a$ and 2b); K14 for the mutation at codon $253(\mathrm{C} \rightarrow \mathrm{T}$ ) (Fig. 2b); and M17, M18, and M26 for the mutation at codon 242 (C $\rightarrow$ T) (Fig. 2b)--showed a low-slope rise but failed to rise. By contrast, the wild-type LTRs of genomic DNA extracted from three BLV-infected cattle (P1-P3) were successfully amplified as well as the positive control (PC), but not negative control (NC), by wild type probe (Fig. 2a and 2b). By contrast, the genomic sample DNA pvAN003 for mutation at codon $252(T \rightarrow C)$ was slightly amplified by the wild type probe with a PVL of 91,566 copies per $10^{5}$ cells (Fig. $2 \mathrm{c}$ and Table 3 ).

Our results clearly showed that three out of the four mutations are present in the probe region of the BLV-CoCoMo-qPCR-2 assay, negatively impacting the sensitivity of BLV-CoCoMo-qPCR-2.

\section{Construction of new three probes to amplify variants with single substitutions at codons 242, 246, and 253}

To amplify three BLV variants, such as M242 (for codon 242), M246 (for codon 246), and M253 (for codon 253), three types of BLV TaqMan probes, designated as p242, p246, and p253, were newly constructed.

When BLV-CoCoMo-qPCR was performed using each single probe and cattle genomic DNAs as shown in Table 3, the amplification curves of each mutant genomic DNA sample was clearly raised (Fig. 2d-f), unlike that in the original BLV-CoCoMo-qPCR-2 performed using a wild type probe (Fig. 2a-b). For example, samples Y14, Y20, K4, K16, K25, T2 and Cb15 were successfully amplified by p246 (Fig. 2d) but not by pWT (Fig. 2a), p242 and p253 (Table 3), with PVLs ranging from 375 to 34,028 copies per $10^{5}$ cells (Table 3). Samples M17, M18, and M26 were amplified by p242 (Fig. 2 e) but not by pWT (Fig. 2 b) and p246 (Table 3), with PVLs ranging from 421 to 44,167 copies per $10^{5}$ cells (Table 3). Sample K14 was amplified by p253 (Fig. 2 f) but not by pWT (Fig. 2 b) and p246 (Table 3), with a PVL of 15,885 copies per $10^{5}$ cells (Table 3 ). In addition, genomic DNA samples extracted from eight BLV-infected cattle carrying wild-type LTR were successfully amplified, with PVLs ranging from 401 to 91,731 copies per $10^{5}$ cells using a wild type probe but not p242, p246, and p253 (Table 3). By contrast, the proviral copy numbers of genomic DNAs extracted from the three BLV-uninfected cattle were estimated to be "0" according to BLV-CoCoMo-qPCR conducted using all four probes (Table 3). Thus, the PVL of each sample could be quantified at various copy numbers, indicating that the three new BLV TaqMan probes are more suitable than the wild type probes for the three mutants M242, M246, and M253.

\section{Development of a new BLV-CoCoMo-qPCR-3 assay using mixed probes}

To develop a new BLV-CoCoMo-qPCR assay that can detect all BLV mutants as well as maintain the original specificity, sensitivity, quantitative nature, and reproducibility, we first examined the optimum ratio of the concentration to be mixed with the wild type (pWT) and three new BLV TaqMan probes ( $p 242$, p246, and p253) using genomic DNAs extracted from three cattle (A11, S38, and S33) infected with the wild-type BLV strain and two cattle (Y20 and K25) infected with M246 variant, which were detected most frequently out of four mutants (Table 4). Four types of mixed probes were used at a ratio as follows: pWT:p242:p246:p253 = (i) 200:33:33:33 ng, (ii) 150:50:50:50 ng, (iii) 100:67:67:67 ng, and (iv) 75:75:75:75 ng. Wild type BLV-infected cattle A11, which had a PVL of 1,237 copies \pm 137 per $10^{5}$ cells as determined using the wild type probe, was successfully detected in all of the triplicate PCR amplifications performed using all four types of mixed probes as well as by only using the wild type probe. By contrast, wild-type BLV-infected cattle S38 and S33, which had PVLs of $<100$ copies per $10^{5}$ cells as determined using the wild type probe, were positive by BLV-CoCoMo-qPCR using mixed probe 200:33:33:33 ng; however, the S38 cattle were negative for one of triplicate PCR amplifications using the other three mixed probes, and S33 was negative for one of the triplicate PCR amplifications using the mixed probe 75:75:75:75 ng. The two BLV-infected cattle (Y20 and K25) carrying the M246 variant were detected by all four types of mixed probes and not only the wild type probe. In addition, the coefficient of variation (CV) of the mixed probe 200:33:33:33 ng ranged between 23.1\% and $3.6 \%$ for BLV PVLs per $10^{5}$ cells of five BLV-infected cattle A11, S38, S33, Y20, and K25, whereas the CVs of three other mixed probes ranged between $74.8 \%$ and $2.8 \%$, indicating 200:33:33:33 ng has less variations than the other three types of mixed probes.

Next, we confirmed whether the mixed probe 200:33:33:33 is suitable for BLV-infected cattle carrying other BLV variants. As predicted, BLV-infected cattle M17, M18, and M26, which have a M242 variant, K14, which have a M253 variant, and pvAN003, which have an M252 variant, were detected in all the triplicate PCR amplifications using the mixed probe 200:33:33:33 ng, and their CVs showed less variation (between 10.9\% and 1.0\%). By contrast, the BLV-uninfected cattle Y1 was estimated to have a value of "O" by BLV-CoCoMo-qPCR using the mixed probe 200:33:33:33 (Table 4).

Thus, based on the new BLV-CoCoMo-qPCR assay performed using a concentration ratio of the four probes, the mixed probe 200:33:33:33 was selected as the optimal probe for the detection of all mutant BLV strains, and this was designated as the "BLV-CoCoMo-qPCR-3" assay.

\section{Reproducibility of the new BLV-CoCoMo-qPCR-3 assay}

To verify the reproducibility of the new BLV-CoCoMo-qPCR-3 assay for the quantification of BLV PVLs, we performed intra- and inter-assays using the genomic DNA from six cattle infected with the wild-type strain and two cattle infected with BLV variants at codon 246 (Table 5). We performed triplicate PCR amplifications for each sample and repeated the same assay thrice to determine the intra-assay reproducibility. The intra-assay CV for BLV PVLs per $10^{5}$ cells 
ranged from $0.4-13.4 \%$ (mean $4.9 \%$ ). We also performed three independent experiments with each sample to determine the inter-assay reproducibility. The inter-assay CV for BLV PVLs per $10^{5}$ cells ranged from 1.5-15.1\% (mean 6.5\%). These results suggest that the intra- and inter-assay reproducibility of the new BLV-CoCoMo-qPCR-3 assay is high.

\section{A comparison of the sensitivity of the new BLV-CoCoMo-qPCR-3 assay with that of the original BLV-CoCoMo-qPCR-2 assay for the detection of the BLV provirus}

We also compared the sensitivity of the new BLV-CoCoMo-qPCR-3 compared to that of BLV-CoCoMo-qPCR-2 for the detection of the BLV provirus using an infectious full-length molecular clone of BLV (pBLV-IF2)[45] (Table 6). We performed a two-fold dilution of the pBLV-IF and adjusted the proviral copy numbers from 100 to 0.1953125 per $10^{5}$ cells in 10 steps and then performed triplicate PCR amplifications and verified the percentage of successful detection. Both methods showed exactly the same detection rate and detected $100 \%(3 / 3)$ of BLV-IFs when present at $\geq 0.78125$ copies, $67 \%(2 / 3)$ when present at 0.390625 copies, and $33 \%(1 / 3)$ when present at 0.1953125 copies. These results indicate that BLV-CoCoMo-qPCR-3 as sensitive and reproducible as the original BLVCoCoMo-qPCR-2 assay in the detection of the low BLV proviral copy numbers.

\section{Evaluation of the sensitivity of the new BLV-CoCoMo-qPCR-3 assay compared with that of the original BLV-CoCoMo-qPCR-2 assay}

We examined the correlation between the quantitative values of the proviral loads using the new BLV-CoCoMo-qPCR-3 and the BLV-CoCoMo-qPCR-2 assay with 100 genomic DNA samples (95 samples were infected with wild-type BLV strain and 5 samples were uninfected). The coefficient of correlation for both methods was 0.99 and the slope was 0.95 (Fig. 3). This result clearly demonstrated that the quantitative values of the new BLV-CoCoMo-qPCR-3 and the BLVCoCoMo-qPCR-2 assay were highly correlated.

\section{Discussion}

Here we developed a new real-time PCR assay that allows the quantification of all mutant BLV strains. Recently, it was reported that some substitutions are present in the nucleobase of the probe region of the BLV-CoCoMo-qPCR-2 assay $[15,39,40]$. If the BLV-CoCoMo-qPCR-2 assay is not improved, it will be impossible to fully detect animals infected with these variants and will lead to the spread of infection within the farm. Thus, improving the BLV-CoCoMo-qPCR2 assay is important to prevent BLV infection or to eradicate BLV as soon as possible. In this study, we found three mutations at codons 246,252 , and 253 located in the probe region of the BLV-CoCoMo-qPCR-2 LTR regions of BLV by the alignment of 206 BLV LTR nucleotide sequences, which were registered in NCBI (January 14, 2020). Based on a nationwide survey conducted in 2011 and 2017, we confirmed that three BLV variants (M242, M246, and M253) are actually present in the probe region of the BLV-CoCoMo-qPCR-2 assay in 11 BLV-infected cattle that were all negative by the BLV-CoCoMo-qPCR assay targeting the BLV LTR gene but positive by the TaKaRa Cycleave PCR assay targeting the BLV tax gene. Thus, this nationwide survey showed that single substitutions in the probe region of the BLV-CoCoMo-qPCR-2 assay were present at a rate of $1.2 \%$ (11 out of 887 samples). Next, we found an M252 variant which was registered in $\mathrm{NCBl}$; however, we could not detect this in the nationwide survey, in a cow sample that was collected from the Tochigi prefecture in another survey conducted in 2009 [39]. Therefore, we investigated the amplification curves of 12 genomic DNAs with a single substitution and clearly showed that three out of four mutations negatively affected the sensitivities of the BLV-CoCoMo-qPCR-2. Thus, we developed a BLV-CoCoMo-qPCR-3 assay that is able to detect various BLV strains having substitutions located in the probe region of BLV-CoCoMo-qPCR-2 and corresponding to nucleotide positions $240-254$ of the LTR regions of BLV.

The BLV-CoCoMo-qPCR-3 assay used a mix of three kind of probes corresponding to three mutations at codons $242(C \rightarrow T), 246(T \rightarrow C)$, and $253(C \rightarrow T)$, excluding one variant (pvAN003) with a substitution at codon $252(T \rightarrow C)$. Because the genomic DNA with only the M252 variant among four variants was clearly amplified by the BLV-CoCoMo-qPCR-2 assay using the wild type probe (Fig. 2c), we did not synthesize a new probe corresponding to this mutation. The nucleobase of $\mathrm{T}$ at codon 252 that is present at the third place from the $3 囚$ side in the probe region of BLV-CoCoMo-qPCR-2 was mutated to C. Because $\mathrm{C}$ is known to have a stronger binding energy than $T$ [46], the nucleobase was mutated from $T$ to $C$, resulting in stronger binding. In addition, since the mutant position is much closer from the $3 \rrbracket$ side of probe, the binding of the probe from the $5 \rrbracket$ side is not affected. This is why this strain could be clearly amplified using the BLV-CoCoMo-qPCR-2 assay.

To create a new BLV-CoCoMo-qPCR assay, the ratio of mixing four types of probes (pWT:p242:p246:p253) was examined. The verification test was conducted by mixing the three types of mutant probes with the wild type probe (pWT:p242:p246:p253) in four different ratios (in ng; 200:33:33:33; 150:50:50:50; 100:67:67:67; and 75:75:75:75) and selected BLV-CoCoMo-qPCR-3 [probe ratio (in ng): 200:33:33:33] as the best assay (Table 4). When the mixing ratios of the probes corresponding to variants were increased, it could not be detected one or two in triplicate PCR amplifications when repeatedly quantified using the genomic DNA (wild type strain) with low proviral loads (Table 4). Our results showed that among the BLV-infected cattle from all over Japan, approximately $99 \%$ were infected with the wild-type strain, whereas only approximately $1 \%$ were infected with the variants mentioned above. Therefore, when making the mixed type of probe, adding a large proportion of the wild-type is important to maintain the sensitivity and reproducibility similar to those of the BLV-CoCoMoqPCR-2 assay, considering the ratio (200:33:33:33) of the four types of probes is optimal in the BLV-CoCoMo-qPCR-3 assay. In addition, we observed sufficient intra- and inter-assay reproducibility of a new BLV-CoCoMo-qPCR-3 assay for the diagnosis using eight infected animals. The assay CV between each sample (0.4-13.4\%) and between each experiment (1.5-15.1\%) (Table 5) was as good as that for the BLV-CoCoMo-qPCR-2 assay (4.8-11.8\%, and 9.0\%, respectively), as shown in previously [26]. Furthermore, this assay showed the same detection sensitivity for provirus (Table 6 ) and high correlation of quantitative nature for PVLs with BLV-CoCoMo-qPCR-2, as shown previously [26] (Fig. 3). Collectively, the BLV-CoCoMo-qPCR-3 assay is a useful tool that enables the simultaneous detection and quantification of the PVLs of all BLV variants.

In general, viruses have been continuously mutating and generating novel strains throughout the evolution process [47]. The mutation rate of retroviruses, such as HIV-1, was estimated to be $1.62 \times 10^{-2}$ nucleotides per site per year [48], whereas that of RSV was estimated to be $1.4 \times 10^{-4}$ nucleotides per 
replication cycle [49] and that of BLV as $9 \times 10^{-5}$ and $3.4 \times 10^{-4}$ nucleotides per year for env gene and LTR, respectively [43]. To achieve persistence through evolution strategies, BLV may be able to avoid immunological surveillance and remain dormant in the infected host [43]. To overcome the possibility of newly emerging variants, it is very important to improve the assay's sensitivity from time to time. Yang et al. (2014) [50] detected several new mutations that were located in the primer and probe regions of real-time PCR assays commonly used for human influenza A viruses and then established a new real-time PCR assay using degenerate nucleotide bases in both the primer and probe, successfully increasing the sensitivity enough to detect new variants of human influenza A viruses. Guillaume et al. (2009) [51] established a single-tube, multiplex, real-time PCR assay using mixed primers and probes to simultaneously detect, differentiate, and quantify the PVLs of HTLV-1, 2, and 3 and simian T-cell leukemia virus types 1 and 3 (STLV-1 and 3). Britta et al (2009) [52] also developed a multiplex real-time PCR assay using mixed primers and probes to simultaneously detect, genotype, and quantify the PVLs of HTLV-1, 2, and 3. Here, it was possible to amplify all BLV strains using degenerate primers designed in our original BLV-CoCoMo-qPCR-2 assay, and the mixed type of probe was used in a new BLV-CoCoMo-qPCR-3 assay. Thus, our method may be useful for detecting severe acute respiratory syndrome coronavirus 2 (SARS-CoV-2), which was initially reported in December 2019 in Wuhan, China [53] and been rapidly spreading around the world, imposing negative consequences for the healthcare system and the overall population. Thus, during the evolution of pathogens in both humans and animals, there is a risk that humans will eventually be affected unless the existing epidemiological and molecular biology techniques are improved from time to time.

BLV infection is causing serious economic damage to the cattle industry. The infection continues to spread gradually as there are no effective treatments or vaccines. It is hoped that BLV infection will be prevented or eradicated from farms as soon as possible. Currently, BLV-CoCoMo-qPCR-3 is considered a highly specific, accurate, and sensitive tool that can detect all BLV strains. We are uncertain of the possibility of new mutations occurring in the nucleobase of the probe region of BLV-CoCoMo-qPCR-3 in the near future. To avoid this possibility, we will discuss degenerate probe synthesis for BLV-CoCoMo-qPCR assay or the development of a new real-time PCR assay targeting different BLV genome sequence regions without mutations.

\section{Conclusion}

We established an improved mixed-probe BLV-CoCoMo-qPCR-3 assay, which successfully amplified all BLV strains, using mixed type of probes to overcome the newly generated mutations located in the probe regions. This method is a useful tool for preventing the spread of BLV infection because it maintains high sensitivity and reproducibility and can detect all mutant strains.

\section{Abbreviations}

Bovine leukemia virus (BLV), human T-leukemia virus types 1 and 2 (HTLV-1 and -2), enzootic bovine leucosis (EBL), proviral load (PVL), coordination of common motifs (CoCoMo), long terminal repeat (LTR), bovine leukocyte antigen (BoLA), fluorescein amidite (FAM), non-fluorescent quencher (NFQ), minor groove binder (MGB), coefficient of variation (CV), and wild type (WT).

\section{Declarations}

\section{Acknowledgments}

The authors thank the veterinary officers of the prefectural Livestock Hygiene Service Centers for their help with blood sampling and the collection of epidemiological data. We also thank Miss. Yiki Matsumoto, Mrs. Mari Kikuya, Miss. Yuka Takahashi, Mrs. Sonoko Abe, Mrs. Toshie Wakamatsu, and other members of the Virus Infectious Disease Field of RIKEN. We are grateful to the Support Unit, Bio-material Analysis, RIKEN BSI Research Resources Center for helping with the sequence analysis. We would like to thank Editage (www.editage.com) for English language editing.

\section{Authors' contributions}

LB and YA conceived and designed the experiments. LB, SY, MI, YS, NT, RY, AY, MM, HM, ST and YA collected samples. LB, SY, MP, HM, ST and YA performed the experiments. LB, SS, ST and YA analyzed the data. YA contributed regents/materials/analysis tools. LB and YA wrote the manuscript. All authors read and approved the final manuscript

\section{Funding}

This research was supported by MAFF-commissioned research "the Strategic Improvement project of the national Surveillance and Diagnosis system for Animal (SISDA)" and by grants from the Projects of the NARO Bio-oriented Technology Research Advancement Institution (the special scheme project on regional developing strategy) (grant number 16817983).

\section{Availability of data and materials}

All data and materials are included in this published article.

\section{Ethics approval}

All animals were handled by veterinarians from the veterinary officers of the prefectural Livestock Hygiene Service Centers, and RIKEN, Japan in strict accordance with good animal practice following the guidelines of RIKEN. The study was approved by the RIKEN Animal Experiments Committee (approval number H29-2-104).

\section{Consent for publication}


Not applicable.

Competing interests

The authors declare that they have no competing interests.

\section{References}

1. Aida Y, Murakami H, Takahashi M, Takeshima SN: Mechanisms of pathogenesis induced by bovine leukemia virus as a model for human T-cell leukemia virus. Front Microbiol 2013, 4:328.

2. Da Y, Shanks RD, Stewart JA, Lewin HA: Milk and fat yields decline in bovine leukemia virus-infected Holstein cattle with persistent lymphocytosis. Proc Natl Acad Sci U S A 1993, 90:6538-6541.

3. Erskine RJ, Bartlett PC, Byrem TM, Render CL, Febvay C, Houseman JT: Association between bovine leukemia virus, production, and population age in Michigan dairy herds. J Dairy Sci 2012, 95:727-734.

4. Ott SL, Johnson R, Wells SJ: Association between bovine-leukosis virus seroprevalence and herd-level productivity on US dairy farms. Prev Vet Med 2003, 61:249-262.

5. Sargeant JM, Kelton DF, Martin SW, Mann ED: Associations between farm management practices, productivity, and bovine leukemia virus infection in Ontario dairy herds. Prev Vet Med 1997, 31:211-221.

6. Vanleeuwen JA, Haddad JP, Dohoo IR, Keefe GP, Tiwari A, Tremblay R: Associations between reproductive performance and seropositivity for bovine leukemia virus, bovine viral-diarrhea virus, Mycobacterium avium subspecies paratuberculosis, and Neospora caninum in Canadian dairy cows. Prev Vet Med 2010, 94:54-64.

7. Konnai S, Murata S, Ohashi K: Immune exhaustion during chronic infections in cattle. J Vet Med Sci 2017, 79:1-5.

8. Nekouei O, VanLeeuwen J, Stryhn H, Kelton D, Keefe G: Lifetime effects of infection with bovine leukemia virus on longevity and milk production of dairy cows. Prev Vet Med 2016, 133:1-9.

9. Kettmann R, Meunier-Rotival M, Cortadas J, Cuny G, Ghysdael J, Mammerickx M, Burny A, Bernardi G: Integration of bovine leukemia virus DNA in the bovine genome. Proc Natl Acad Sci U S A 1979, 76:4822-4826.

10. Tajima S, Ikawa Y, Aida Y: Complete bovine leukemia virus (BLV) provirus is conserved in BLV-infected cattle throughout the course of B-cell lymphosarcoma development. J Virol 1998, 72:7569-7576.

11. Ohno A, Takeshima SN, Matsumoto Y, Aida Y: Risk factors associated with increased bovine leukemia virus proviral load in infected cattle in Japan from 2012 to 2014. Virus Res 2015, 210:283-290.

12. Tajima S, Takahashi M, Takeshima SN, Konnai S, Yin SA, Watarai S, Tanaka Y, Onuma M, Okada K, Aida Y: A mutant form of the tax protein of bovine leukemia virus (BLV), with enhanced transactivation activity, increases expression and propagation of BLV in vitro but not in vivo. $J$ Viro/ $2003,77: 1894$ 1903.

13. Moe KK, Polat M, Borjigin L, Matsuura R, Hein ST, Moe HH, Aida Y: New evidence of bovine leukemia virus circulating in Myanmar cattle through epidemiological and molecular characterization. PLOS ONE 2020, 15:e0229126.

14. Polat M, Ohno A, Takeshima SN, Kim J, Kikuya M, Matsumoto Y, Mingala CN, Onuma M, Aida Y: Detection and molecular characterization of bovine leukemia virus in Philippine cattle. Arch Virol 2015, 160:285-296.

15. Polat M, Takeshima SN, Hosomichi K, Kim J, Miyasaka T, Yamada K, Arainga M, Murakami T, Matsumoto Y, de la Barra Diaz V, et al: A new genotype of bovine leukemia virus in South America identified by NGS-based whole genome sequencing and molecular evolutionary genetic analysis. Retrovirology 2016, 13:4.

16. Polat M, Moe HH, Shimogiri T, Moe KK, Takeshima SN, Aida Y: The molecular epidemiological study of bovine leukemia virus infection in Myanmar cattle. Arch Virol 2017, 162:425-437.

17. Jimba M, Takeshima SN, Matoba K, Endoh D, Aida Y: BLV-CoCoMo-qPCR: Quantitation of bovine leukemia virus proviral load using the CoCoMo algorithm. Retrovirology 2010, 7:91.

18. Sato H, Watanuki S, Murakami H, Sato R, Ishizaki H, Aida Y: Development of a luminescence syncytium induction assay (LuSIA) for easily detecting and quantitatively measuring bovine leukemia virus infection. Arch Virol 2018, 163:1519-1530.

19. Kobayashi T, Inagaki Y, Ohnuki N, Sato R, Murakami S, Imakawa K: Increasing Bovine leukemia virus (BLV) proviral load is a risk factor for progression of Enzootic bovine leucosis: A prospective study in Japan. Prev Vet Med 2019.

20. Somura Y, Sugiyama E, Fujikawa H, Murakami K: Comparison of the copy numbers of bovine leukemia virus in the lymph nodes of cattle with enzootic bovine leukosis and cattle with latent infection. Arch Virol 2014, 159:2693-2697.

21. Mekata H, Sekiguchi S, Konnai S, Kirino Y, Honkawa K, Nonaka N, Horii Y, Norimine J: Evaluation of the natural perinatal transmission of bovine leukaemia virus. Vet Rec $2015,176: 254$

22. Mekata H, Sekiguchi S, Konnai S, Kirino Y, Horii Y, Norimine J: Horizontal transmission and phylogenetic analysis of bovine leukemia virus in two districts of Miyazaki, Japan. J Vet Med Sci 2015, 77:1115-1120.

23. Yuan Y, Kitamura-Muramatsu Y, Saito S, Ishizaki H, Nakano M, Haga S, Matoba K, Ohno A, Murakami H, Takeshima SN, Aida Y: Detection of the BLV provirus from nasal secretion and saliva samples using BLV-CoCoMo-qPCR-2: Comparison with blood samples from the same cattle. Virus Res 2015, 210:248-254. 
24. Watanuki S, Takeshima SN, Borjigin L, Sato H, Bai L, Murakami H, Sato R, Ishizaki H, Matsumoto Y, Aida Y: Visualizing bovine leukemia virus (BLV)infected cells and measuring BLV proviral loads in the milk of BLV seropositive dams. Vet Res 2019, 50:102.

25. Lo CW, Borjigin L, Saito S, Fukunaga K, Saitou E, Okazaki K, Mizutani T, Wada S, Takeshima SN, Aida Y: BoLA-DRB3 Polymorphism is Associated with Differential Susceptibility to Bovine Leukemia Virus-Induced Lymphoma and Proviral Load. Viruses 2020, 12:352.

26. Takeshima SN, Kitamura-Muramatsu Y, Yuan Y, Polat M, Saito S, Aida Y: BLV-CoCoMo-qPCR-2: improvements to the BLV-CoCoMo-qPCR assay for bovine leukemia virus by reducing primer degeneracy and constructing an optimal standard curve. Arch Virol 2015, 160:1325-1332.

27. Juliarena MA, Barrios CN, Ceriani MC, Esteban EN: Hot topic: Bovine leukemia virus (BLV)-infected cows with low proviral load are not a source of infection for BLV-free cattle. J Dairy Sci 2016, 99:4586-4589.

28. Jimba M, Takeshima SN, Murakami H, Kohara J, Kobayashi N, Matsuhashi T, Ohmori T, Nunoya T, Aida Y: BLV-CoCoMo-qPCR: a useful tool for evaluating bovine leukemia virus infection status. BMC Vet Res 2012, 8:167.

29. Bai L, Yokoyama K, Watanuki S, Ishizaki H, Takeshima SN, Aida Y: Development of a new recombinant p24 ELISA system for diagnosis of bovine leukemia virus in serum and milk. Arch Virol 2019, 164:201-211.

30. Benitez OJ, Roberts JN, Norby B, Bartlett PC, Maeroff JE, Grooms DL: Lack of Bovine leukemia virus transmission during natural breeding of cattle. Theriogenology 2019, 126:187-190.

31. Benitez OJ, Roberts JN, Norby B, Bartlett PC, Takeshima SN, Watanuki S, Aida Y, Grooms DL: Breeding bulls as a potential source of bovine leukemia virus transmission in beef herds. J Am Vet Med Assoc 2019, 254:1335-1340.

32. Panei CJ, Takeshima SN, Omori T, Nunoya T, Davis WC, Ishizaki H, Matoba K, Aida Y: Estimation of bovine leukemia virus (BLV) proviral load harbored by lymphocyte subpopulations in BLV-infected cattle at the subclinical stage of enzootic bovine leucosis using BLV-CoCoMo-qPCR. BMC Vet Res 2013, 9:95.

33. Sato H, Watanuki S, Bai L, Borjigin L, Ishizaki H, Matsumoto $Y$, Hachiya $Y$, Sentsui $H$, Aida $Y$ : A sensitive luminescence syncytium induction assay (LuSIA) based on a reporter plasmid containing a mutation in the glucocorticoid response element in the long terminal repeat U3 region of bovine leukemia virus. Virol J 2019, 16:66.

34. Miyasaka T, Takeshima SN, Sentsui H, Aida Y: Identification and diversity of bovine major histocompatibility complex class II haplotypes in Japanese Black and Holstein cattle in Japan. J Dairy Sci 2012, 95:420-431.

35. Takeshima SN, Ohno A, Aida Y: Bovine leukemia virus proviral load is more strongly associated with bovine major histocompatibility complex class II DRB3 polymorphism than with DQA1 polymorphism in Holstein cow in Japan. Retrovirology 2019, 16:14.

36. Takeshima SN, Sasaki S, Meripet P, Sugimoto Y, Aida Y: Single nucleotide polymorphisms in the bovine MHC region of Japanese Black cattle are associated with bovine leukemia virus proviral load. Retrovirology 2017, 14:24.

37. Hamada R, Metwally S, Polat M, Borjigin L, Ali A, Abdel-Hady A, Mohamed A, Wada S, Aida Y: Detection and molecular characterization of bovine leukemia virus in Egyptian dairy cattle. Front Vet Sci 7: 608 doi: 103389/fvets 2020.

38. Assi W, Hirose T, Wada S, Matsuura R, Takeshima S-n, Aida Y: PRMT5 Is Required for Bovine Leukemia Virus Infection In Vivo and Regulates BLV Gene Expression, Syncytium Formation, and Glycosylation In Vitro. Viruses 2020, 12:650.

39. Murakami H, Uchiyama J, Suzuki C, Nikaido S, Shibuya K, Sato R, Maeda Y, Tomioka M, Takeshima SN, Kato H, et al: Variations in the viral genome and biological properties of bovine leukemia virus wild-type strains. Virus Res 2018, 253:103-111.

40. Pluta A, Rola-Luszczak M, Douville RN, Kuzmak J: Bovine leukemia virus long terminal repeat variability: identification of single nucleotide polymorphisms in regulatory sequences. Virol $\mathrm{J} 2018,15: 165$.

41. Rovnak J, Boyd AL, Casey JW, Gonda MA, Jensen WA, Cockerell GL: Pathogenicity of molecularly cloned bovine leukemia virus. J Viro/ 1993, 67:70967105 .

42. Mansky LM, Temin HM: Lower mutation rate of bovine leukemia virus relative to that of spleen necrosis virus. J Viro/ 1994, 68:494-499.

43. Willems L, Kerkhofs P, Burny A, Mammerickx M, Kettmann R: Lack of LTR and ENV genetic variation during bovine leukemia virus-induced leukemogenesis. Virology 1995, 206:769-772.

44. Murakami H, Todaka H, Uchiyama J, Sato R, Sogawa K, Sakaguchi M, Tsukamoto K: A point mutation to the long terminal repeat of bovine leukemia virus related to viral productivity and transmissibility. Virology 2019, 537:45-52.

45. Matsuura R, Inabe K, Otsuki H, Kurokawa K, Dohmae N, Aida Y: Three YXXL Sequences of a Bovine Leukemia Virus Transmembrane Protein are Independently Required for Fusion Activity by Controlling Expression on the Cell Membrane. Viruses 2019, 11:1140.

46. Abbas H: First-principles study of interaction of serine with nucleobases of DNA and RNA. J Biol Phys 2017, 43:105-111.

47. Lloyd-Smith JO, George D, Pepin KM, Pitzer VE, Pulliam JR, Dobson AP, Hudson PJ, Grenfell BT: Epidemic dynamics at the human-animal interface. Science 2009, 326:1362-1367.

48. Fu YX: Estimating mutation rate and generation time from longitudinal samples of DNA sequences. Mol Biol Evol 2001, 18:620-626.

49. Leider JM, Palese P, Smith Fl: Determination of the mutation rate of a retrovirus. J Viro/ 1988, 62:3084-3091.

50. Yang JR, Kuo CY, Huang HY, Wu FT, Huang YL, Cheng CY, Su YT, Chang FY, Wu HS, Liu MT: Newly emerging mutations in the matrix genes of the human influenza $A(H 1 N 1) p d m 09$ and $A(H 3 N 2)$ viruses reduce the detection sensitivity of real-time reverse transcription-PCR. J Clin Microbio/ $2014,52: 76-82$.

51. Besson G, Kazanji M: One-step, multiplex, real-time PCR assay with molecular beacon probes for simultaneous detection, differentiation, and quantification of human T-cell leukemia virus types 1, 2, and 3. J Clin Microbio/ 2009, 47:1129-1135.

52. Moens B, Lopez G, Adaui V, Gonzalez E, Kerremans L, Clark D, Verdonck K, Gotuzzo E, Vanham G, Cassar O, et al: Development and validation of a multiplex real-time PCR assay for simultaneous genotyping and human T-lymphotropic virus type 1, 2, and 3 proviral load determination. $J$ Clin Microbiol 
2009, 47:3682-3691.

53. Wu Z, McGoogan JM: Characteristics of and Important Lessons From the Coronavirus Disease 2019 (COVID-19) Outbreak in China: Summary of a Report of 72314 Cases From the Chinese Center for Disease Control and Prevention. JAMA 2020, 323:1239-1242.

\section{Tables}

Table 1

Detection of BLV provirus with BLV-CoCoMo-qPCR-2 and TaKaRa-Cycleave qPCR assays.

\begin{tabular}{|c|c|c|c|c|c|c|c|c|c|c|c|c|c|c|c|c|c|c|c|c|c|c|c|c|}
\hline & \multicolumn{22}{|c|}{2011} & \multirow{2}{*}{$\begin{array}{l}2017 \\
\text { Tochig }\end{array}$} \\
\hline & & \multicolumn{2}{|c|}{ Okinawa } & \multicolumn{2}{|l|}{ Mie } & \multicolumn{2}{|c|}{ Shiga } & \multicolumn{2}{|l|}{ Gifu } & \multicolumn{2}{|c|}{ Ishigawa } & \multicolumn{2}{|c|}{ Shizuoka } & \multicolumn{2}{|c|}{ Kanagawa } & \multicolumn{2}{|c|}{ Chiba } & \multicolumn{2}{|c|}{ Niigata } & \multicolumn{2}{|c|}{ Yamagata } & \multicolumn{2}{|c|}{ Miyagi } & \\
\hline Farms & & a & $b$ & a & $\mathrm{b}$ & a & $b$ & a & b & a & $b$ & a & $\mathrm{b}$ & a & $b$ & a & $b$ & a & b & a & $b$ & a & b & a \\
\hline Cattle No. & & 20 & 30 & 30 & 20 & 30 & 20 & 30 & 20 & 30 & 20 & 33 & 30 & 30 & 30 & 30 & 19 & 30 & 20 & 30 & 20 & 30 & 20 & 30 \\
\hline $\begin{array}{l}\text { BLV- } \\
\text { CoCoMo- }\end{array}$ & $+^{c}$ & 23 & 18 & 24 & 5 & 20 & 12 & 17 & 18 & 29 & 3 & 11 & 4 & 14 & 26 & 28 & 11 & 23 & 16 & 25 & 9 & 20 & 20 & 16 \\
\hline qPCR-2 ${ }^{\mathrm{a}}$ & - & 7 & 2 & 6 & 15 & 10 & 8 & 13 & 2 & 1 & 17 & 22 & 26 & 16 & 4 & 2 & 8 & 7 & 4 & 5 & 11 & 10 & 0 & 14 \\
\hline Takara- & + & 20 & 18 & 27 & 5 & 19 & 11 & 17 & 18 & 27 & 4 & 11 & 4 & 15 & 25 & 28 & 11 & 23 & 15 & 21 & 9 & 16 & 16 & 17 \\
\hline $\mathrm{qPCR}^{\mathrm{b}}$ & - & 10 & 2 & 3 & 15 & 11 & 9 & 13 & 2 & 3 & 16 & 22 & 26 & 15 & 5 & 2 & 8 & 7 & 5 & 9 & 11 & 14 & 4 & 13 \\
\hline
\end{tabular}

${ }^{a}$ BLV-CoCoMo-qPCR-2 (Takeshima et al., 2015) was used to detect for the BLV LTR genes. ${ }^{\text {b }}$ Cycleave PCR BLV detection kit (TaKaRa Bio inc.) was used to detect for the BLV tax gene. " "+" and "-" represent the judgment results by two assays are positive and negative, respectively.

Table 2

Comparison of the detection of BLV provirus with the BLV-CoCoMo-qPCR-2 and Takara-Cycleave PCR assays.

\begin{tabular}{|lll|}
\hline \multicolumn{2}{|c|}{ Detection of BLV provirus } & Samples No. / Total No. \\
BLV-CoCoMo-qPCR-2 $^{\text {a }}$ & Takara-cycleave PPCR $^{\mathrm{b}}$ & \\
$\boldsymbol{+}^{\mathrm{c}}$ & + & $557 / 887$ \\
- & + & $11 / 887$ \\
\hline+ & - & $33 / 887$ \\
\hline- & - & $286 / 887$ \\
\hline
\end{tabular}

${ }^{\text {a }}$ BLV-CoCoMo-qPCR-2 (Takeshima et al., 2015) was used to detect for the BLV LTR genes. ${ }^{\text {b }}$ Cycleave PCR BLV detection kit (TaKaRa Bio inc.) was used to detect for the BLV tax gene. " "+" and "-" represent the judgment results by two assays are positive and negative, respectively. 
Table 3

Quantification of the BLV proviral load with the BLV-CoCoMo-qPCR assay using different types of probes.

\begin{tabular}{|c|c|c|c|c|c|}
\hline \multirow[t]{2}{*}{ BLV mutant ${ }^{a}$} & \multirow[t]{2}{*}{ Samples ID } & \multicolumn{4}{|c|}{ PVL quantified by CoCoMo-qPCR with four types of probes } \\
\hline & & pWT & p242 & p246 & p253 \\
\hline \multirow[t]{3}{*}{ M242: CTIAGCTCTCGGTCC } & M18 & 0 & 44167 & 0 & nt \\
\hline & M26 & 0 & 1009 & 0 & nt \\
\hline & M17 & 0 & 421 & 0 & nt \\
\hline \multirow[t]{7}{*}{ 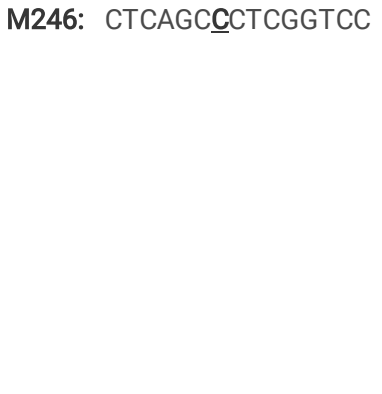 } & Y14 & 0 & 0 & 34028 & 0 \\
\hline & $\mathrm{T} 2$ & 0 & 0 & 30604 & 0 \\
\hline & Y20 & 0 & 0 & 26220 & 0 \\
\hline & K16 & 0 & 0 & 22500 & 0 \\
\hline & K4 & 0 & 0 & 20000 & 0 \\
\hline & K25 & 0 & 0 & 5943 & 0 \\
\hline & Cb15 & 0 & $\mathrm{nt}^{\mathrm{c}}$ & 375 & nt \\
\hline M252: CTCAGCTCTCGGㄷCC & pvAN003 & 91566 & nt & $\mathrm{nt}$ & nt \\
\hline M253: CTCAGCTCTCGGTIC & K14 & 0 & $\mathrm{nt}$ & 0 & 15885 \\
\hline \multirow[t]{8}{*}{ WT: CTCAGCTCTCGGTCC } & T43 & 91731 & 0 & 0 & 0 \\
\hline & T41 & 41754 & 0 & 0 & 0 \\
\hline & K7 & 21667 & 0 & 0 & 0 \\
\hline & K4 & 15855 & 0 & 0 & 0 \\
\hline & $\mathrm{Ta} 2$ & 13962 & 0 & 0 & 0 \\
\hline & $\mathrm{Ka} 2$ & 5566 & 0 & 0 & 0 \\
\hline & T1 & 1182 & 0 & 0 & 0 \\
\hline & T112 & 401 & 0 & 0 & 0 \\
\hline \multirow[t]{3}{*}{ Uninfected } & $\mathrm{K} 1$ & 0 & 0 & 0 & 0 \\
\hline & K9 & 0 & 0 & 0 & 0 \\
\hline & T51 & 0 & 0 & 0 & 0 \\
\hline
\end{tabular}

${ }^{a}$ M242, M246, M252, and M253 represent the four types of single mutation at codons $242(C \rightarrow T), 246(T \rightarrow C), 252(T \rightarrow C)$ and $253(C \rightarrow T)$ in the probe region between residue 240 and 254 of the BLV-CoCoMo-qPCR-2 in the BLV LTR genes, respectively. ${ }^{b}$ pWT, p242, p246, and p253 represent the wild type (WT) of probe and three new types of probes designed for each type of BLV variants (M242, M246, and M253). PVL represent the BLV proviral loads in $10^{5}$ cells. ${ }^{\mathrm{c}} \mathrm{nt}$, not test. 
Table 4

Quantification of the BLV proviral load with the BLV-CoCoMo-qPCR assay using wild and mixed probes.

\begin{tabular}{|c|c|c|c|c|c|c|c|c|c|c|c|c|c|c|c|}
\hline \multicolumn{2}{|c|}{ Ratio, conc. (ng) } & \multirow{2}{*}{\multicolumn{3}{|c|}{$\begin{array}{l}\text { pWT } \\
300 \mathrm{ng}\end{array}$}} & \multicolumn{11}{|c|}{ pWT: p246: p242: p253c } \\
\hline \multirow[b]{2}{*}{ LTR $^{a}$} & \multirow[b]{2}{*}{ Sample } & & & & \multicolumn{3}{|c|}{ 200: 33: 33: 33 ng } & \multicolumn{3}{|c|}{ 150: 50: $50: 50 \mathrm{ng}$} & \multicolumn{3}{|c|}{ 100: 67: 67: $67 \mathrm{ng}$} & \multicolumn{2}{|c|}{ 75: 75: $75: 75 \mathrm{ng}$} \\
\hline & & PVL & $\begin{array}{l}\text { Avg. } \\
\pm S D\end{array}$ & $\mathrm{CV}$ & $P V L^{b}$ & $\begin{array}{l}\text { Avg. } \\
\pm S D\end{array}$ & $\mathrm{CV}$ & PVL & $\begin{array}{l}\text { Avg. } \\
\pm S D\end{array}$ & $\mathrm{CV}$ & $P V L$ & $\begin{array}{l}\text { Avg. } \\
\pm S D\end{array}$ & $\mathrm{CV}$ & $P V L$ & $\begin{array}{l}\text { Avg. } \\
\pm S D\end{array}$ \\
\hline \multirow[t]{9}{*}{ WT } & \multirow[t]{3}{*}{$\mathrm{A} 11$} & 1079 & & & 1202 & & & 1298 & & & 1297 & & & 1459 & \\
\hline & & 1316 & $\begin{array}{l}1237 \\
\pm \\
137\end{array}$ & 9.0 & 1202 & $\begin{array}{l}1260 \pm \\
101\end{array}$ & 6.6 & 1316 & $\begin{array}{l}1342 \\
\pm 61\end{array}$ & 3.7 & 1604 & $\begin{array}{l}1441 \\
\pm 154\end{array}$ & 8.7 & 1604 & $\begin{array}{l}1462 \\
\pm 140\end{array}$ \\
\hline & & 1316 & & & 1377 & & & 1412 & & & 1423 & & & 1324 & \\
\hline & \multirow[t]{3}{*}{ S33 } & 69 & & & 77 & & & 100 & & & 150 & & & 98 & \\
\hline & & 57 & $\begin{array}{l}72 \pm \\
17\end{array}$ & 18.9 & 94 & $83 \pm 10$ & 9.4 & 58 & $\begin{array}{l}78 \pm \\
21\end{array}$ & 22.1 & 125 & $\begin{array}{l}91 \pm \\
80\end{array}$ & 71.6 & 93 & $\begin{array}{l}97 \pm \\
3.3\end{array}$ \\
\hline & & 90 & & & 78 & & & 76 & & & 0 & & & 100 & \\
\hline & \multirow[t]{3}{*}{ S38 } & 24 & & & 67 & & & 0 & & & 94 & & & 76 & \\
\hline & & 26 & $\begin{array}{l}33 \pm \\
11\end{array}$ & 33.9 & 39 & $51 \pm 15$ & 23.1 & 59 & $\begin{array}{l}41 \pm \\
36\end{array}$ & 70.9 & 0 & $\begin{array}{l}57 \pm \\
50\end{array}$ & 71.8 & 51 & $\begin{array}{l}42 \pm \\
39\end{array}$ \\
\hline & & 49 & & & 47 & & & 65 & & & 76 & & & 0 & \\
\hline \multirow[t]{6}{*}{ M246 } & \multirow[t]{3}{*}{ Y20 } & 0 & & & 24444 & & & 24537 & & & 25596 & & & 27248 & \\
\hline & & 0 & $0 \pm 0$ & 0.0 & 26296 & $\begin{array}{l}25741 \\
\pm 1226\end{array}$ & 3.6 & 26574 & $\begin{array}{l}25679 \\
\pm \\
1040\end{array}$ & 3.3 & 24679 & $\begin{array}{l}25627 \\
\pm 964\end{array}$ & 3.1 & 24037 & $\begin{array}{l}24893 \\
\pm \\
2064\end{array}$ \\
\hline & & 0 & & & 26481 & & & 25926 & & & 26606 & & & 23394 & \\
\hline & \multirow[t]{3}{*}{ K25 } & 0 & & & 5283 & & & 4874 & & & 5171 & & & 4886 & \\
\hline & & 0 & $0 \pm 0$ & 0.0 & 4874 & $\begin{array}{l}5157 \pm \\
246\end{array}$ & 3.9 & 5236 & $\begin{array}{l}5110 \\
\pm 205\end{array}$ & 3.3 & 5423 & $\begin{array}{l}5184 \\
\pm 232\end{array}$ & 3.7 & 5203 & $\begin{array}{l}4962 \\
\pm 214\end{array}$ \\
\hline & & 0 & & & 5315 & & & 5220 & & & 4959 & & & 4797 & \\
\hline \multirow[t]{9}{*}{ M242 } & \multirow[t]{3}{*}{ M18 } & & & & 53539 & & & & & & & & & & \\
\hline & & nt & & & 52584 & $\begin{array}{l}51236 \\
\pm 3198\end{array}$ & 6.2 & nt & & & nt & & & $\mathrm{nt}$ & \\
\hline & & & & & 47584 & & & & & & & & & & \\
\hline & \multirow[t]{3}{*}{ M26 } & & & & 1078 & & & & & & & & & & \\
\hline & & nt & & & 990 & $\begin{array}{l}1013 \pm \\
57\end{array}$ & 5.7 & nt & & & nt & & & nt & \\
\hline & & & & & 971 & & & & & & & & & & \\
\hline & \multirow[t]{3}{*}{ M17 } & & & & 403 & & & & & & & & & & \\
\hline & & nt & & & 382 & $\begin{array}{l}379 \pm \\
26\end{array}$ & 6.8 & nt & & & nt & & & nt & \\
\hline & & & & & 351 & & & & & & & & & & \\
\hline \multirow[t]{3}{*}{ M252 } & \multirow[t]{3}{*}{ pvAN003 } & & & & 104828 & & & & & & & & & & \\
\hline & & nt & & & 105517 & $\begin{array}{l}104598 \\
\pm 1053\end{array}$ & 1.0 & nt & & & nt & & & nt & \\
\hline & & & & & 103448 & & & & & & & & & & \\
\hline \multirow[t]{3}{*}{ M253 } & K14 & & & & 15973 & & & & & & & & & & \\
\hline & & nt & & & 15537 & $\begin{array}{l}14832 \\
\pm 1613\end{array}$ & 10.9 & nt & & & nt & & & nt & \\
\hline & & & & & 12987 & & & & & & & & & & \\
\hline $\mathrm{Neg}$ & Y1 & & & & 0 & & & & & & & & & & \\
\hline & & nt & & & 0 & $0 \pm 0$ & 0.0 & nt & & & nt & & & nt & \\
\hline & & & & & 0 & & & & & & & & & & \\
\hline
\end{tabular}


a LTR represent long terminal repeated genes of BLV; WT (wild type), M242, M246, M252 and M253 represent no mutation and single mutation codons 242 $(C \rightarrow T), 246(T \rightarrow C), 252(T \rightarrow C)$ and $253(C \rightarrow T)$ in the probe region of the BLV-CoCoMo-qPCR-2 in the LTR genes, respectively. Neg represent the cattle uninfected (negative) by BLV. ${ }^{b}$ PVL represent proviral loads in $10^{5}$ cells from triplicate PCR amplifications from each sample; Avg represent standard deviation; SD represent the average PVL; CV represent coefficient of variation between each experiment. nt represent not test. ${ }^{\mathrm{c}} \mathrm{pWT}$, p242, p246 and p253 represent the wild type (WT) of probe and three new types of probes designed for each type of mutant (M242, M246 and M253).

Table 5

Intra- and inter-assay reproducibility of the BLV-CoCoMo-qPCR-3 assay using probe mixed at ratio of 200:33:33:33

\begin{tabular}{|c|c|c|c|c|c|c|c|c|c|}
\hline \multirow{2}{*}{\multicolumn{3}{|c|}{$\begin{array}{l}\text { PVL by } \\
\text { pWT or p246 (300 ng) }\end{array}$}} & \multicolumn{7}{|c|}{ PVL by pWT: p242: p246: p253bه200: 33: 33: $33 \mathrm{ng}$} \\
\hline & & & \multirow{2}{*}{$\begin{array}{c}\text { Exp.1 } \\
\text { Avg. } \pm S D\end{array}$} & \multirow{2}{*}{$\begin{array}{c}\text { Exp.2 } \\
\text { Avg. } \pm S D\end{array}$} & \multirow{2}{*}{$\begin{array}{c}\text { Exp.3 } \\
\text { Avg. } \pm S D\end{array}$} & \multicolumn{3}{|c|}{ Intra-assay ${ }^{d}$} & \multirow{2}{*}{$\begin{array}{l}\text { Inter assaye } \\
\text { Exp. } 1 \llbracket 3\end{array}$} \\
\hline LTR $^{a}$ & ID & Avg. $\pm \mathrm{SD}^{\mathrm{c}}$ & & & & Exp.1 & Exp.2 & Exp.3 & \\
\hline \multirow[t]{6}{*}{ WT } & A10 & $20222 \pm 329$ & $20736 \pm 3391$ & $19065 \pm 1345$ & $18903 \pm 1605$ & 13.4 & 5.8 & 6.9 & 4.2 \\
\hline & A25 & $10046 \pm 413$ & $9801 \pm 414$ & $9851 \pm 224$ & $9520 \pm 358$ & 3.4 & 1.9 & 3.1 & 1.5 \\
\hline & T71 & $6684 \pm 146$ & $5806 \pm 222$ & $5479 \pm 242$ & $5534 \pm 244$ & 3.1 & 3.6 & 3.6 & 2.6 \\
\hline & S48 & $610 \pm 136$ & $698 \pm 94$ & $669 \pm 10$ & $544 \pm 51$ & 11.0 & 1.2 & 7.7 & 10.5 \\
\hline & s9 & $n t^{f}$ & $368 \pm 2$ & $366 \pm 29$ & $416 \pm 58$ & 0.4 & 6.5 & 11.4 & 6.1 \\
\hline & S33 & $148 \pm 20$ & $83 \pm 8$ & $63 \pm 2$ & $91 \pm 6$ & 9.4 & 2.7 & 6.3 & 15.1 \\
\hline \multirow[t]{2}{*}{ M246 } & Y20 & $27535 \pm 379$ & $23578 \pm 1456$ & $25413 \pm 734$ & $27453 \pm 555$ & 5.0 & 2.4 & 1.7 & 6.2 \\
\hline & K25 & nt & $6141 \pm 109$ & $5081 \pm 166$ & $5688 \pm 177$ & 1.5 & 2.7 & 2.5 & 5.6 \\
\hline
\end{tabular}

a LTR represent long terminal repeated genes of BLV; WT (wild type) and M246 represent the no mutation and single mutation at codon 246 ( $\rightarrow C$ ) in the probe region between residue 240 and 254 of the BLV-CoCoMo-qPCR-2 in the BLV LTR genes, respectively.

${ }^{b}$ pWT, p242, p246 and p253 represent the wild type (WT) of probe and three new types of probes designed for each type of mutant (M242, M246 and M253). ${ }^{\text {c }}$ Values represent the average \pm standard deviation (SD) of BLV proviral loads (PVL) in $10^{5}$ cells from triplicate PCR amplifications from each sample. ${ }^{d}$ Intra$\mathrm{CV}$ : Coefficient of variation between each sample. ${ }^{\mathrm{e}}$ Inter-CV: Coefficient of variation between each experiment. ${ }^{\mathrm{f}} \mathrm{nt}$ represent not test.

Table 6

Comparison between BLV proviral detection with the original BLV-CoCoMo-qPCR-2 assay and the new BLV-CoCoMo-qPCR-

\begin{tabular}{|lll|}
\hline $\begin{array}{l}\text { BLV (pBLV-IF2) } \\
\text { (copy numbers) }\end{array}$ & CoCoMo-qPCR-2 ${ }^{\mathrm{a}}$ & CoCoMo-qPCR-3 $^{\mathrm{b}}$ \\
\hline 100 & $3 / 3^{\mathrm{c}}$ & $3 / 3$ \\
\hline 50 & $3 / 3$ & $3 / 3$ \\
\hline 25 & $3 / 3$ & $3 / 3$ \\
12.5 & $3 / 3$ & $3 / 3$ \\
\hline 6.25 & $3 / 3$ & $3 / 3$ \\
\hline 3.125 & $3 / 3$ & $3 / 3$ \\
\hline 1.5625 & $3 / 3$ & $3 / 3$ \\
\hline 0.78125 & $3 / 3$ & $3 / 3$ \\
\hline 0.390625 & $2 / 3$ & $2 / 3$ \\
\hline 0.1953125 & $1 / 3$ & $1 / 3$ \\
\hline 0 & $0 / 3$ & $0 / 3$ \\
\hline
\end{tabular}

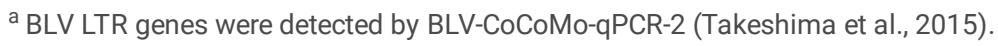

${ }^{b}$ BLV LTR genes were detected by BLV-CoCoMo-qPCR-3. ${ }^{c}$ Number detected per number tested. 


\section{6}

$242 \quad 246 \quad 252253$

$240 \mathbf{7} \quad 260$

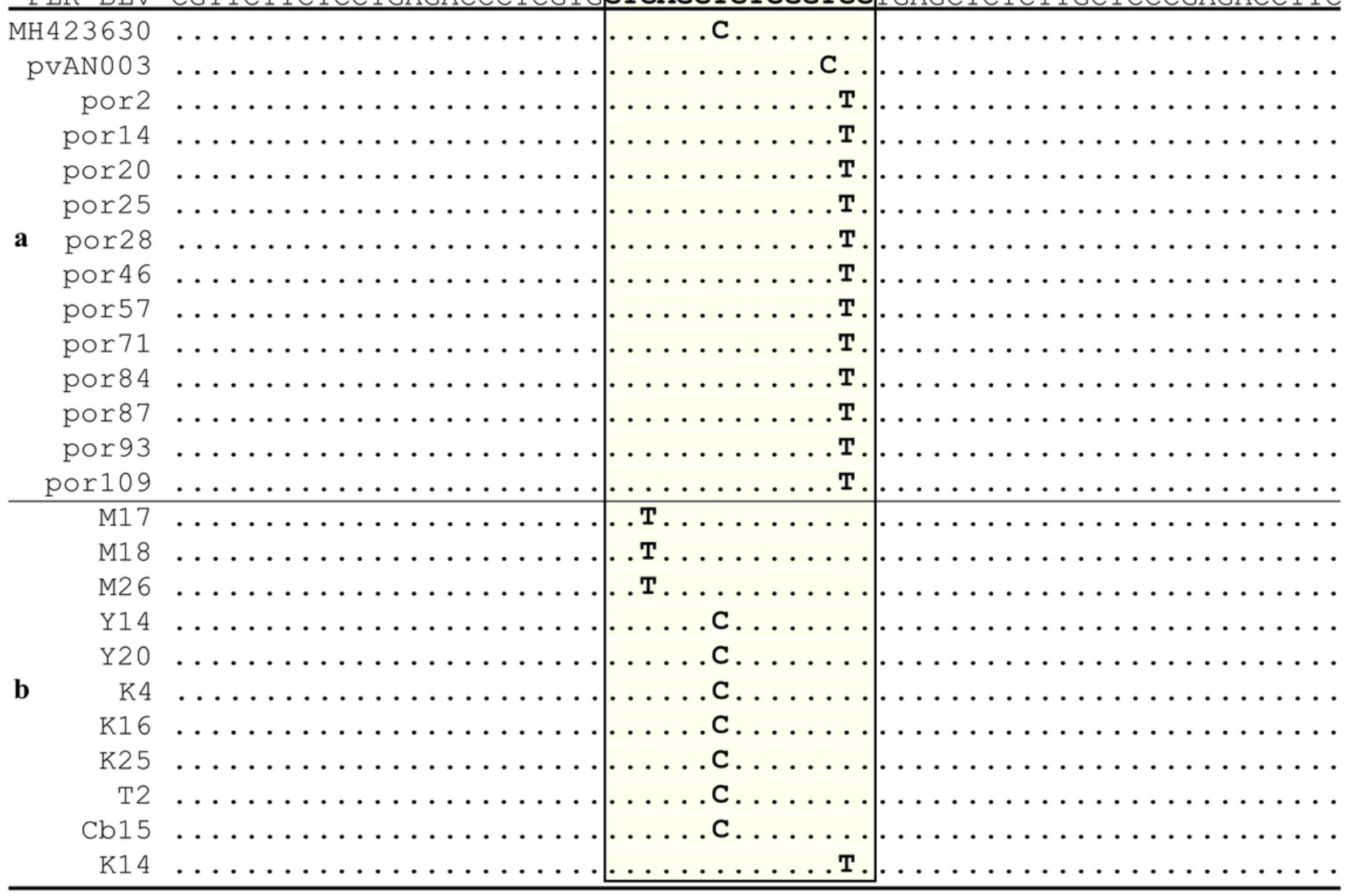

Probe region of CoCoMo-qPCR-2

\section{Figure 1}

Alignment of partial nucleotide sequences of BLV LTR genes from a total 14 strains in GenBank. (a) A total of 11 strains were detected in this nationwide survey conducted in 2011 and 2017 (b). BLV isolates from this nationwide survey sample were submitted to GenBank [accession numbers: LC532162 (M17), LC585344 (M18), LC585346 (M26), LC585348 (Y14), LC585349 (Y20), LC585345 (K4), LC532161 (K16), LC585347 (K25), LC585350 (T2), LC585351 (Cb15), and LC585352 (K14)]. (a) Representation of three types of single mutations at codons $246(T \rightarrow C), 252(T \rightarrow C)$, and $253(C \rightarrow T)$ in the probe region between the residue 240 and 254 of the BLV-CoCoMo-qPCR-2 in the BLV LTR genes from the 14 BLV LTR nucleotide sequences from GenBank. (b) Representation of the three types of single mutations at codons $242(C \rightarrow T), 246(T \rightarrow C)$, and $253(C \rightarrow T)$ in the probe region between residue 240 and 254 of the BLV-CoCoMo-qPCR2 in the BLV LTR genes in the 11 BLV LTR nucleotide sequences in this nationwide survey. Dots indicate identity with FLK-BLV strain pBLV913 (accession number: EF600696) [41]. 


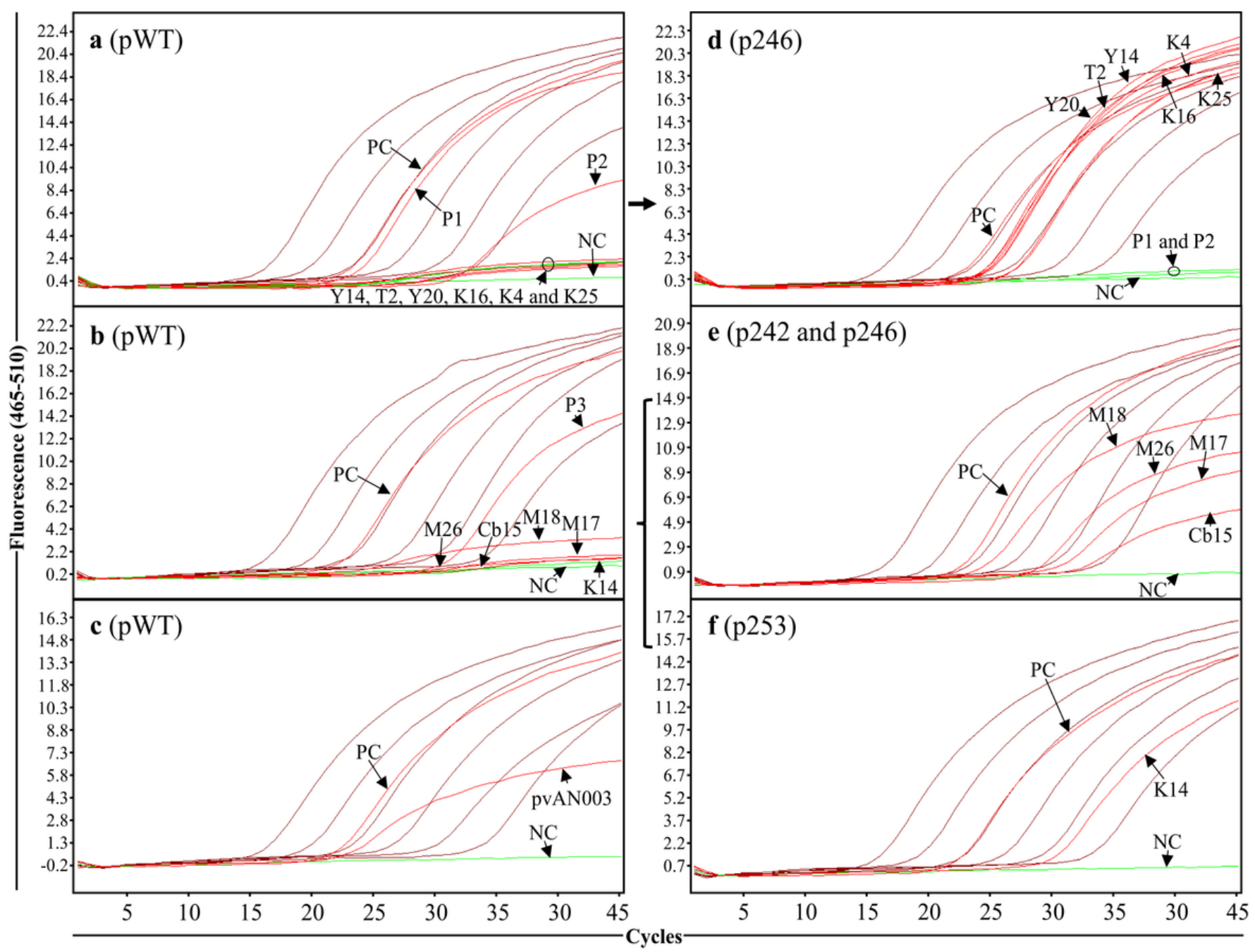

Figure 2

Amplification curves of cattle genomic DNA by BLV-CoCoMo-qPCR assays using wild-type and three new BLV TaqMan probes. (a-c) The results by CoCoMoqPCR-2 using wild type probe, and (d-f) the results by CoCoMo-qPCR using one of the three new probes p242, p246, and p253 corresponding to each mutant strain. The shapes of the amplification curves were different between genomic DNAs that were extracted from BLV-infected cattle, with wild-type and variants with four types of single mutation at codons $242(C \rightarrow T), 246(T \rightarrow C), 252(T \rightarrow C)$, and $253(C \rightarrow T)$ in the probe region of the BLV-CoCoMo-qPCR-2. P1, P2, and $\mathrm{P} 3$ were infected with the wild-type strain, whereas $\mathrm{M} 17, \mathrm{M} 18$, and M26 were infected with the strain with single mutation at codons $242(\mathrm{C} \rightarrow \mathrm{T})$ and $\mathrm{Y} 14, \mathrm{Y} 20$, $\mathrm{K} 4, \mathrm{~K} 16, \mathrm{~K} 25, \mathrm{~T} 2$ and $\mathrm{Cb} 15$ were infected with the strain with single mutations at codons $246(\mathrm{~T} \rightarrow \mathrm{C})$. pvAN003 was infected with the strain with single mutation at codons $252(\mathrm{~T} \rightarrow \mathrm{C})$, and K14 was infected with the strain with single mutation at codons $253(\mathrm{C} \rightarrow \mathrm{T})$. PC and NC were the positive and negative control, respectively. 


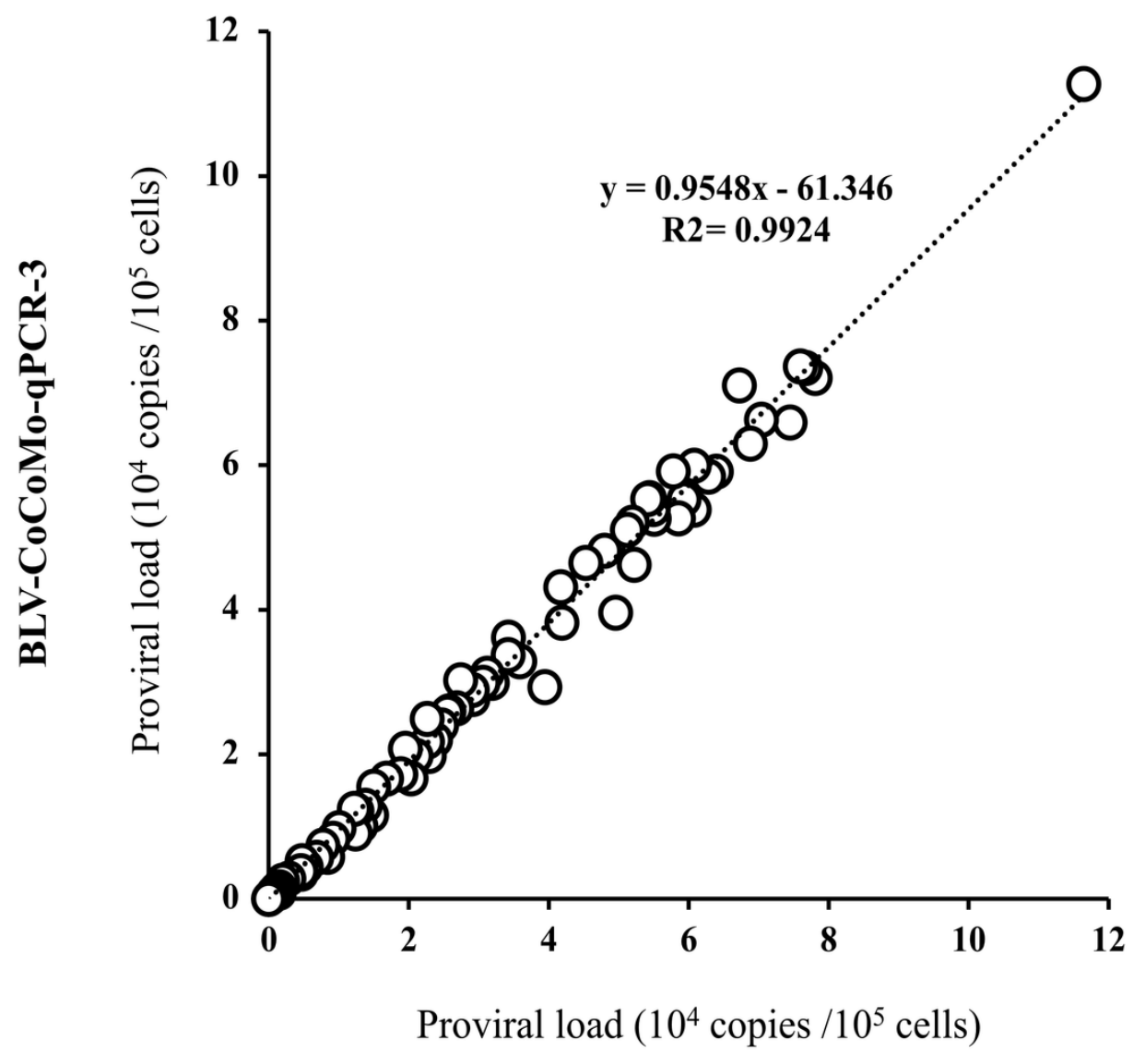

\section{BLV-CoCoMo-qPCR-2}

Figure 3

Correlation between the proviral loads of 100 samples that were judged using the original BLV-CoCoMo-qPCR-2 with a wild type probe and the new BLVCoCoMo-qPCR-3 assay using a mix of four probes. 